\title{
Universal Influenza Vaccines: Shifting To Better Vaccines
}

Francesco Berlanda Scorza $^{1^{*}}$, Vadim Tsvetnitsky $^{1,2}$, John J. Donnelly $^{1}$

${ }^{1}$ PATH: 2201 Westlake Avenue Suite 200 Seattle, WA 98121 USA

${ }^{2}$ Present address:International AIDS Vaccine Initiative 125 Broad Street, 9th Floor New York, NY 10004

*Corresponding authorfberlanda-scorza@path.org

Keywords: influenza vaccines, universal vaccines, chimeric hemagglutinin, heterosubtypic immunity

\section{Funding And Conflict Of Interest Statement}

Funding from the Bill \& Melinda Gates Foundation supported PATH staff members in their work on this manuscript. The corresponding author at PATH collaborates with the Icahn School of Medicine at Mount Sinai on the development of a chimeric HA universal influenza vaccine.

\section{Acknowledgements}

The authors thank Lauren Newhouse and Lisa Anderson for their valuable editorial and support services. 


\begin{abstract}
Influenza virus causes acute upper and lower respiratory infections and is the most likely, among known pathogens, to cause a large epidemic in humans. Influenza virus mutates rapidly, enabling it to evade natural and vaccine-induced immunity. Furthermore, influenza viruses can cross from animals to humans, generating novel, potentially pandemic strains. Currently available influenza vaccines induce a strain specific response and may be ineffective against new influenza viruses. The difficulty in predicting circulating strains has frequently resulted in mismatch between the annual vaccine and circulating viruses. Low-resource countries remain mostly unprotected against seasonal influenza and are particularly vulnerable to future pandemics, in part, because investments in vaccine manufacturing and stockpiling are concentrated in high-resource countries. Antibodies that target conserved sites in the hemagglutinin stalk have been isolated from humans and shown to confer protection in animal models, suggesting that broadly protective immunity may be possible. Several innovative influenza vaccine candidates are currently in preclinical or early clinical development. New technologies include adjuvants, synthetic peptides, virus-like particles (VLPs), DNA vectors, messenger RNA, viral vectors, and attenuated or inactivated influenza viruses. Other approaches target the conserved exposed epitope of the surface exposed membrane matrix protein M2e. Well-conserved influenza proteins, such as nucleoprotein and matrix protein, are mainly targeted for developing strong cross-protective T cell responses. With multiple vaccine candidates moving along the testing and development pipeline, the field is steadily moving toward a product that is more potent, durable, and broadly protective than previously licensed vaccines.
\end{abstract}


Influenza viruses circulate globally and affect all age groups. Vaccines are the best tools available for preventing influenza illness and are recommended annually by the World Health Organization (WHO) for groups at high risk of mortality or significant morbidity, including pregnant women, children six months to five years of age, elderly individuals (65 years of age and older), individuals with chronic medical conditions and health care workers. The US Centers for Disease Control and Prevention (CDC) have broadened the WHO recommendation for influenza vaccination to all persons older than six months of age, with two doses for children between six months and eight years old who are receiving influenza vaccine for the first time.

Human influenza viruses are classified into three types (A, B, C) based on the following highly conserved internal proteins: matrix protein 1 (M1), membrane matrix protein (M2), and nucleoprotein (NP). Type A influenza viruses are further sub-divided into subtypes based on the antigenicity of their hemagglutinin (HA) and neuraminidase (NA) surface glycoproteins. Currently, 18 HA and 9 NA subtypes of influenza A are known and all exist in aquatic birds, their natural reservoirs. Influenza B viruses infect only humans, but two antigenically and phylogenetically distinct lineages co-circulate. Type $\mathrm{C}$ influenza viruses are known to infect humans and pigs but infections are rare. Species tropism, particularly of influenza A, is determined by the presence, anatomic location and structure of terminal sialic acid residues that mediate viral attachment and entry. Influenza's antigenic variation, which has limited the development of a broadly protective vaccine, manifests as either antigenic drift or antigenic shift. Antigenic drift is the ability of the virus to escape pre-existing immunity through point mutations in the genes encoding HA and NA, making circulating strain prediction difficult and antigenic mismatch likely. Antigenic shift is defined by the recombination of HA genes that results in the generation of a novel influenza A strain to which a large proportion of the human population has no pre-existing immunity, therefore increasing the potential for a pandemic, as occurred during the shift from $\mathrm{H} 1$ to H2 in 1957 and $\mathrm{H} 2$ to $\mathrm{H} 3$ in 1968.

The 2009 influenza A/H1N1 pandemic (a new strain of A/H1N1), the highly pathogenic avian influenza 
strains (A/H5N1 and A/H7N9), and past experiences with seasonal influenza vaccine mismatch have all illustrated the unpredictability of the virus and the challenges to mounting a global response against newly emerging virus strains. Currently, influenza A H1 and H3 subtypes co-circulate. The direct transmission of highly pathogenic avian strains to humans (such as influenza A/H5N1 and, more recently, influenza A/H7N9) presents an additional threat, but such strains have not yet demonstrated the capacity

for efficient transmission in humans. Vaccination remains the preferred approach toward controlling influenza; however, the challenge of annual large-scale immunizations is currently beyond the capacities of low-resource countries, which need to improve vaccine delivery across all age groups, with a special focus on children. For such immunizations to become a reality, a vaccine that induces broadly crossprotective and durable immunity would needto be developed.

\section{Licensed Influenza Vaccines And Their Limitations}

In general, influenza vaccines have an excellent safety record, but their efficacy varies significantly in various age groups and against different strains. Rare exceptions to the safety profile has emerged in association to the use of specific adjuvants, as discussed later. Strain mismatch and pandemics are frequent causes for vaccine failure and, over the last few years, several mismatches and one pandemic have occurred. The development and deployment of an H1N1 pdm2009 vaccine was relatively rapid, but the vaccine still became available too late to provide timely protection to vulnerable populations during the pandemic (Fig. 1, yellow line). Vaccine mismatch with seasonal influenza strains also has occurred multiple times, for example during the 2003/2004 season when an A/Fujian/411/2002 virus emerged as a new and unanticipated antigenic variant of influenza A/H3N2(Fig. 1, orange line). Of 326 influenza A/H3N2 isolates characterized, only 25 percent were antigenically similar to the widely used vaccine strain A/Panama/2007/99 (H3N2). During the 2007/2008 influenza season, A/Wisconsin/67/2005 (H3N2) was included in the vaccine, but A/Brisbane/10/2007 (H3N2) was the dominant circulating strain, resulting in a particularly severe influenza season (Fig. 1, grey line). A mismatch of the vaccine to the recent 2012/2013 influenza season resulted in only 46\% efficacy in adults 18 through 49 years and $9 \%$ 
efficacy in people older than 65 years of age(Fig. 1, green line). In this case, the circulating strain prediction was correct, but the mismatch was caused by a mutation in the egg-adapted seed virus (IVR165) that was sent to vaccine manufacturers. The mutation was not present in the WHO-recommended strain but occurred during strain adaptation to growth in eggs.

Influenza vaccine mismatch also occurred during the 2014/2015 influenza season (Fig. 1, brown line). The influenza A/H3N2 strain included in the vaccine (A/Texas/50/2012) was selected based on the most common circulating influenza $\mathrm{A} / \mathrm{H} 3 \mathrm{~N} 2$ virus in February 2014. The drifted influenza A/Switzerland/9715293/2013 virus was first detected in the United States in March 2014 in a small number of samples. Before the end of the year, it became the prevalent circulating strain and the vaccine available in the fall of 2014 offered poor protection against the mismatch, with an efficacy estimated around $18 \%$ for influenza $\mathrm{A} / \mathrm{H} 3 \mathrm{~N} 2$. As a result, the influenza-associated hospitalization rate among people 65 years of age and older in the 2014/2015 season was the highest recorded since the CDC began tracking those data in 2005. In February 2015, WHO recommended that the new A/Switzerland/9715293/2013 (H3N2)-like virus to be included in the vaccine for the 2015/2016 season.

In the case of live-attenuated influenza vaccines (LAIVs), the efficacy of Flumist ${ }^{\circledR}$ in randomized clinical trials was tempered by limited effectiveness against influenza A (H1N1) pdm09 in children during the 2013/2014 influenza season. These results may be specific to the influenza A/H1N1 component of the vaccine since the original $\mathrm{A} / \mathrm{California}$ strain has been found to be more susceptible to thermal degradation due to a unique HA stalk sequence [1]. The full reason for its lack of effectiveness, however, is not entirely understood and is the subject of ongoing investigation. The past and current seasons exemplify the unpredictability of seasonal influenza epidemics and the challenge for current-generation vaccines that can only follow changes in circulating viruses. They also highlight opportunities for a universal vaccine that would induce broadly protective immunity. 


\section{General Approaches For Low- And Middle-Income Country (LMIC) Markets}

While availability of data on disease burden in LIMIC remains limited,a recent study has shown that, during year 2008, seasonal influenza was associated globally with $2-7 \%$ of deaths from acute lower respiratory infections(ALRI) inchildrenyounger than 5 years and resulted in 28,000-111,500 deaths, of which $99 \%$ occurred in developing countries. Influenza A, particularly H3N2 subtype, caused higher morbidity and mortality than influenza B[2]. As for pandemic outbreaks, models based on the 1918-1920 influenza pandemic estimate that up to 62 million people could die if a similar pandemic occurred today, and approximately $96 \%$ of the deaths would occur in the developing world [3]. Despite these figures, investments for improved seasonal and pandemic vaccine manufacturing and stockpiles are concentrated in high-resource countries. More than $80 \%$ of seasonal influenza vaccine doses produced between 2009 and 2010 originated from seven large manufacturers located in the United States, Canada, Australia, western Europe, Russia, China, and Japan. Moreover, no pandemic influenza A/H1N1 vaccine was available in the majority of low-resource countries before January 2010, more than eight months after the WHO declared a pandemic. Many low-resource countries do not have a full picture of their influenza disease burden due to limited surveillance. In some tropical settings, influenza circulates year-round, making influenza-related morbidity substantial, especially as a major contributor to childhood pneumonia [4].

Five companies currently offer WHO-prequalified split virion inactivated trivalent or quadrivalent seasonal influenza vaccines (GlaxoSmithKline, Green Cross Corp., Hualan Bio, Novartis, and Sanofi Pasteur) or seasonal trivalent LAIV (Serum Institute of India). Several companies (CSL, Green Cross Corp., MedImmune, Novartis, Sanofi Pasteur and Serum Institute of India) obtained prequalification of their pandemic influenza vaccines only for the 2009 pandemic influenza A/H1N1 virus. Despite sufficient influenza vaccine production capacity for annual needs in high-resource countries, influenza vaccination coverage rates among high-risk groups are below the targets set by national governments and recommended by WHO in these settings. In LMICs, differing health priorities and constraints on health 
budgets primarily limit influenza vaccine use to private markets, with the possible exception of PanAmerican Health Organization countries. Expanding influenza vaccine coverage to public markets in LMICs is unlikely to happen in the absence of data from cost-benefit studies. Local production is expected to reduce cost and improve availability of vaccines in some LMICs, but the lack of local recommendations and government funding for vaccine use remains a limiting factor. Limited regional manufacturing capacity for seasonal vaccines also results in limited capacity to respond to pandemic outbreaks. The use of LAIVs may contribute to improved coverage rates in children since they cost less than IIVs to produce and administer, particularly if the age indication for current LAIV, currently two years of age, can be safely extended to a younger population.

\section{Technical And Regulatory Assessment}

Regulatory agencies recognize hemagglutination inhibition (HAI) titers as the primary correlate of protection and of influenza vaccine efficacy. In the case of LAIVs, the correlate of protection for universal vaccines might not be linked with hemagglutination inhibiting antibodies. Anti-stalk antibodies do not inhibit hemagglutination. Vaccine approaches based on viral proteins other than HA would also not affect hemagglutination. These factors pose a higher regulatory barrier. In the absence of animmune correlate, efficacy against a clinically meaningful endpoint will have to be demonstrated. The existing paradigm for currently licensed influenza vaccines is to define protection as the vaccine's ability to prevent illness associated with documented influenza infection. This is distinct from a vaccine's ability to reduce disease severity, which may be an important component of vaccine-mediated protection and may depend on both humoral and cellular immunity. Addressing a reduction in disease severity can be a worthwhile strategy in the development of universal or broadly reactive vaccines, but no influenza vaccine has been licensed so far on the basis of prevention of severe disease.

Despite the importance of animal models in the development of broadly reactive vaccines, carefully controlled human studies are essential. Field efficacy trials are costly and subject to a variety of 
confounding factors and biases. Human challenge studies allow for detailed measurements of the kinetics and magnitude of a range of immune responses, which can help with the development of correlates of protection. Wild-type influenza viruses have been used for challenge studies in adults, while attenuated vaccine strains of influenza have been used to perform challenge studies in children. In many instances however, the results of these studies have been difficult to interpret. In some instances, volunteers have been protected from influenza illness despite the lack of a measurable immune response to vaccination, although a possible explanation could be that disease was subclinical or not all non-vaccinated volunteers were reliably infected.

\section{Status Of Influenza Vaccine Research And Development Activities}

New technologies currently in development include faster generation of reassortant seed viruses, improved manufacturing consistency and yield, use of cell culture instead of eggs, improved adjuvants, quadrivalent vaccines, and prime-boost strategies. Other innovative approaches aim at inducing immune responses to less immuno-dominant epitopes, a phenomenon termed 'unnatural immunity' [5]. Vaccine candidates currently in the development pipeline (Table 1) can be divided into the following general categories: 1) those designed to elicit antibody responses to structurally conserved regions of HA and surface exposed membrane matrix protein (M2e), and 2) those that induce cross-protective T-cell responses against internal proteins like NP and M1. The former type of immunogens are intended to prevent infection while the latter are meant to reduce disease severity. Approaches targeting a crossprotective T-cell response against conserved internal influenza proteins such as NP and M1 are geared toward inducing a reduction in disease severity and could be valuable both for priming and boosting an immune response.

The HA molecule comprises two major structural elements, the head and stalk regions. The head is immuno-dominant and the primary target for currently licensed influenza vaccines.HAs are phylogenetically divided into group 1 (H1, H2, H5, H6, H8, H9, H11, H12, H13, H16, H17, and H18) and 
group $2(\mathrm{H} 3, \mathrm{H} 4, \mathrm{H} 7, \mathrm{H} 10, \mathrm{H} 14$, and H15) based on similarities in their stalk regions. With some exceptions, the head portion of the HA molecule is highly variable and protection by neutralizing antibodies targeting this region is limited to the same strain or subtype. On the other hand, monoclonal antibodies against conserved epitopes on the stalk recognize multiple subtypes. A murine monoclonal antibody, CR9114, has been shown to bind the HA stalk and protect against lethal challenge with influenza A and B [6]. Recently, a neutralizing antibody has been identified in humans that recognizes a conserved portion of the HA glycoprotein and neutralizes all 18 subtypes of both group 1 and group 2 influenza A viruses [7]. The challenge will be to develop an immunogen capable of inducing such antibodies.

Novel approaches have been undertaken to focus the immune responses against the HA stalk. In the first approach, a "headless" HA is designed by introducing a deletion in the HA sequence.Multiple approaches have recently been published to stabilize the headless stem. In one paper, researchers introduced mutations to stabilize the core of the hemagglutinin stem and bound these modified hemagglutinin to ferritin nanoparticles[8]. A second group applied a combination of mutations that realigned the subunits of the stem at the top resulting in soluble trimeric HA (mini-HAs) [9]. When the teams vaccinated mice, both groups saw full protection against H5N1. The nanoparticle-anchoring vaccine showed partial protection in ferrets, whereas the other vaccine showed partial protection in nonhuman primates. Future work will include extending these technologies to additional strains. Since viruses with a headless HA would be replication incompetent, this type of approach is limited to recombinant proteins, virus-like particles (VLPs), or nucleic acid vaccines.

The second approach used to overcome the sub-dominance of stalk epitopes is to induce anti-stalk antibodies via immunization with antigenically-distant viruses. One strategy is to vaccinate sequentially with chimeric HA constructs having the same stalk but heads derived from different strains. Sequential exposure to the same stalk domain and divergent exotic head domains has been shown in animal models 
to boost anti-stalk cross-reactive antibodies. The induction of stalk-reactive antibodies has been demonstrated in humans after influenza A/H5N1 vaccine administration. Adults have pre-existing immunity against H1 (group $1 \mathrm{HA}$ ) and immunization with influenza A/H5N1 vaccine (also group $1 \mathrm{HA}$ ) induces high titers of stalk-reactive antibodies that are cross-protective in passive transfer experiments in mice [10].

Additional strategies to develop HA-based universal vaccines include Computationally Optimized Broadly Reactive Antigen (COBRA) from consensus HA sequences; HA peptides linked to the Ii-Key moiety of the class 2 Major Histocompatibility Complex (Antigen Express, Inc.); and fusion constructs with other proteins or immune-stimulant molecules (Table 1). Other influenza virus proteins could also be used to develop a universal vaccine. The influenza A virus ion channel M2 is expressed at high density on the cell membrane of virus-infected cells and at low density in the lipid membrane of the mature influenza virus. M2 has a small, non-glycosylated 24 amino acid ectodomain (M2e) that is highly conserved among human influenza A viruses. While natural infection does not usually induce an immune response to M2e in humans, antibodies can bind to M2e expressed on virus-infected host cells and reduce virus replication in experimental models. Since a robust M2e response might require the use of an adjuvant to induce high antibody titers, several M2e vaccine candidates are currently in clinical development as proteins conjugated to immune-stimulatory molecules to adjuvant the immune response [11]. Unfortunately, an M2e-based vaccine is unlikely to cover influenza B because its M2e comprises only four amino acids and is too small to be an effective immunogen.

Antibodies against NA interfere with virus release from the cell surface, effectively reducing the amount of infectious virus produced by infected cells. Two NA subtypes, N1 and N2, are commonly found in humans and NA, similar to HA, undergoes antigenic drift within a virus subtype. The majority of current seasonal vaccines include NA but its amount is not standardized, raising concerns that vaccines with low NA content may not induce NA antibodies at high enough titers to confer protection. Recent studies have 
shown that animals immunized with adjuvanted NA are protected against homologous and heterologous viral challenge, though within the same virus subtype [12]. Both NP and M1 proteins are well conserved in influenza A viruses. The stimulation of T cell-based immunity against those targets is considered an important aspect of the development of broadly reactive vaccines (Table 1). T cell-based immunity, however, is not expected to prevent infection, only to modulate it by reducing viral shedding and disease severity.

Adjuvants such as oil-in-water emulsions MF59 and ASO3 have been shown to increase the number of recognized HA epitopes and, consequently, to increase cross-recognition of non-matched strains. This was mainly demonstrated for pandemic influenza A/H5N1 vaccines which generally do not elicit robust cross-protection against multiple virus strains. MF59 in seasonal vaccines has thus far been well tolerated and has induced more potent, durable, and broader immune responses in children than non-adjuvanted vaccines. When evaluated in adult and elderly populations, MF59 has been shown to improve the immunogenicity of an influenza A/H5N1 vaccine. Notably, MF59 also boosted protection against influenza virus strains not fully matched to those included in the vaccine, as in the case of the A/Fujian/2002 H3N3 mismatch in 2003/2004 [13]. Other oil-in-water adjuvants used in influenza vaccines include AS03, although safety concerns have been raised after the increased incidence of narcolepsy and risk of anaphylaxis following administration of the 2009 AS03-adjuvanted monovalent pandemic A/H1N1 vaccine [14][15].

\section{Summary}

No consensus has been achieved on what primary clinical endpoint universal influenza vaccines should achieve to be considered successful. Whether or not they should completely prevent influenza infection or reduce the severity of disease is still being debated. A truly universal influenza vaccine would offer a combination of protection from antigenic drift and shift and ideally confer lifelong immunity. Adjuvants have been moderately effective in increasing cross-reactivity against viral strains not included in the 
vaccine. Several novel technologies focused on innovative immunogens are currently in preclinical and early clinical development. While the influenza field has seen substantial investment over the last ten years, the majority of funding has focused on ensuring the sustainability of manufacturing within high resource settings and broadening manufacturing options so that influenza vaccines are not solely dependent on egg-based production. In recent years, however, investments have been increasing in the development of novel approaches to immunogen design, offering the potential for significant improvements in the performance of future influenza vaccines. 
Figure 1. Percentage Of Patient Visits For Influenza-Like Illness In The Us For Selected Seasons And Years Of Mismatch.

Scale represents percentage of patient visits for influenza-like illness for selected seasons discussed in the text. Data are from the US Centers for Disease Control and Prevention's US Outpatient Influenza-like Illness Surveillance Network. Data for 2014-2015 are through week 10, 2015. 


\section{References}

[1] Cotter CR, Jin H, Chen Z. A single amino acid in the stalk region of the H1N1pdm influenza virus HA protein affects viral fusion, stability and infectivity. PLoS Pathog 2014;10:e1003831. doi:10.1371/journal.ppat.1003831.

[2] Nair H, Brooks WA, Katz M, Roca A, Berkley JA, Madhi SA, et al. Global burden of respiratory infections due to seasonal influenza in young children: a systematic review and meta-analysis. Lancet (London, England) 2011;378:1917-30. doi:10.1016/S0140-6736(11)61051-9.

[3] Murray CJL, Lopez AD, Chin B, Feehan D, Hill KH. Estimation of potential global pandemic influenza mortality on the basis of vital registry data from the 1918-20 pandemic: a quantitative analysis. Lancet 2006;368:2211-8. doi:10.1016/S0140-6736(06)69895-4.

[4] Brooks WA, Goswami D, Rahman M, Nahar K, Fry AM, Balish A, et al. Influenza is a major contributor to childhood pneumonia in a tropical developing country. Pediatr Infect Dis J 2010;29:216-21. doi:10.1097/INF.0b013e3181bc23fd.

[5] Nabel GJ, Fauci AS. Induction of unnatural immunity: prospects for a broadly protective universal influenza vaccine. Nat Med 2010;16:1389-91. doi:10.1038/nm1210-1389.

[6] Dreyfus C, Laursen NS, Kwaks T, Zuijdgeest D, Khayat R, Ekiert DC, et al. Highly conserved protective epitopes on influenza B viruses. Science 2012;337:1343-8. doi:10.1126/science. 1222908 .

[7] Corti D, Voss J, Gamblin SJ, Codoni G, Macagno A, Jarrossay D, et al. A neutralizing antibody selected from plasma cells that binds to group 1 and group 2 influenza A hemagglutinins. Science 2011;333:850-6. doi:10.1126/science.1205669.

[8] Yassine HM, Boyington JC, McTamney PM, Wei C-J, Kanekiyo M, Kong W-P, et al. Hemagglutinin-stem nanoparticles generate heterosubtypic influenza protection. Nat Med 2015;21:1065-70. doi:10.1038/nm.3927. 
[9] Impagliazzo A, Milder F, Kuipers H, Wagner M, Zhu X, Hoffman RMB, et al. A stable trimeric influenza hemagglutinin stem as a broadly protective immunogen. Science (80- ) 2015;349:13016. doi:10.1126/science.aac7263.

[10] Krammer F, Jul-Larsen A, Margine I, Hirsh A, Sjursen H, Zambon M, et al. An H7N1 Influenza Virus Vaccine Induces Broadly Reactive Antibody Responses against H7N9 in Humans. Clin Vaccine Immunol 2014;21:1153-63. doi:10.1128/CVI.00272-14.

[11] Turley CB, Rupp RE, Johnson C, Taylor DN, Wolfson J, Tussey L, et al. Safety and immunogenicity of a recombinant M2e-flagellin influenza vaccine (STF2.4xM2e) in healthy adults. Vaccine 2011;29:5145-52. doi:10.1016/j.vaccine.2011.05.041.

[12] Wohlbold TJ, Nachbagauer R, Xu H, Tan GS, Hirsh A, Brokstad KA, et al. Vaccination with adjuvanted recombinant neuraminidase induces broad heterologous, but not heterosubtypic, crossprotection against influenza virus infection in mice. MBio 2015;6:e2556. doi:10.1128/mBio.02556-14.

[13] GIUDiCE G, HILBERT A, BUGARINI R, MinUtello A, POPOVA O, TONEATTO D, Et Al. An MF59-adjuvanted inactivated influenza vaccine containing A/Panama/1999 (H3N2) induced broader serological protection against heterovariant influenza virus strain A/Fujian/2002 than a subunit and a split influenza vaccine. Vaccine 2006;24:3063-5. doi:10.1016/j.vaccine.2006.01.015.

[14] Nohynek H, Jokinen J, Partinen M, Vaarala O, Kirjavainen T, Sundman J, et al. AS03 adjuvanted AH1N1 vaccine associated with an abrupt increase in the incidence of childhood narcolepsy in Finland. PLoS One 2012;7:e33536. doi:10.1371/journal.pone.0033536.

[15] Rouleau I, De Serres G, Drolet JP, Skowronski DM, Ouakki M, Toth E, et al. Increased risk of anaphylaxis following administration of 2009 AS03-adjuvanted monovalent pandemic A/H1N1 (H1N1pdm09) vaccine. Vaccine 2013;31:5989-96. doi:10.1016/j.vaccine.2013.10.033. 
[16] Hekele A, Bertholet S, Archer J, Gibson DG, Palladino G, Brito LA, et al. Rapidly produced SAM ${ }^{\circledR}$ vaccine against H7N9 influenza is immunogenic in mice. Emerg Microbes Infect 2013;2:e52. doi:10.1038/emi.2013.54.

[17] Langley JM, Frenette L, Ferguson L, Riff D, Sheldon E, Risi G, et al. Safety and cross-reactive immunogenicity of candidate AS03-adjuvanted prepandemic H5N1 influenza vaccines: a randomized controlled phase 1/2 trial in adults. J Infect Dis 2010;201:1644-53. doi:10.1086/652701.

[18] Krammer F, Palese P, Steel J. Advances in universal influenza virus vaccine design and antibody mediated therapies based on conserved regions of the hemagglutinin. Curr Top Microbiol Immunol 2015;386:301-21. doi:10.1007/82_2014_408.

[19] Cummings JF, Guerrero ML, Moon JE, Waterman P, Nielsen RK, Jefferson S, et al. Safety and immunogenicity of a plant-produced recombinant monomer hemagglutinin-based influenza vaccine derived from influenza A (H1N1)pdm09 virus: a Phase 1 dose-escalation study in healthy adults. Vaccine 2014;32:2251-9. doi:10.1016/j.vaccine.2013.10.017.

[20] Atsmon J, Caraco Y, Ziv-Sefer S, Shaikevich D, Abramov E, Volokhov I, et al. Priming by a novel universal influenza vaccine (Multimeric-001)-a gateway for improving immune response in the elderly population. Vaccine 2014;32:5816-23. doi:10.1016/j.vaccine.2014.08.031.

[21] Stoloff GA, Caparros-Wanderley W. Synthetic multi-epitope peptides identified in silico induce protective immunity against multiple influenza serotypes. Eur J Immunol 2007;37:2441-9. doi:10.1002/eji.200737254.

[22] Mössler C, Groiss F, Wolzt M, Wolschek M, Seipelt J, Muster T. Phase I/II trial of a replicationdeficient trivalent influenza virus vaccine lacking NS1. Vaccine 2013;31:6194-200. doi:10.1016/j.vaccine.2013.10.061.

[23] De Filette M, Martens W, Smet A, Schotsaert M, Birkett A, Londoño-Arcila P, et al. Universal influenza A M2e-HBc vaccine protects against disease even in the presence of pre-existing antiHBc antibodies. Vaccine 2008;26:6503-7. doi:10.1016/j.vaccine.2008.09.038. 
[24] Yan J, Villarreal DO, Racine T, Chu JS, Walters JN, Morrow MP, et al. Protective immunity to H7N9 influenza viruses elicited by synthetic DNA vaccine. Vaccine 2014;32:2833-42. doi:10.1016/j.vaccine.2014.02.038.

[25] Lambe T, Carey JB, Li Y, Spencer AJ, van Laarhoven A, Mullarkey CE, et al. Immunity against heterosubtypic influenza virus induced by adenovirus and MVA expressing nucleoprotein and matrix protein-1. Sci Rep 2013;3:1443. doi:10.1038/srep01443.

[26] Kanekiyo M, Wei C-J, Yassine HM, McTamney PM, Boyington JC, Whittle JRR, et al. Selfassembling influenza nanoparticle vaccines elicit broadly neutralizing H1N1 antibodies. Nature 2013;499:102-6. doi:10.1038/nature12202.

[27] Altenburg AF, Kreijtz JHCM, de Vries RD, Song F, Fux R, Rimmelzwaan GF, et al. Modified vaccinia virus ankara (MVA) as production platform for vaccines against influenza and other viral respiratory diseases. Viruses 2014;6:2735-61. doi:10.3390/v6072735.

[28] Schmitz N, Beerli RR, Bauer M, Jegerlehner A, Dietmeier K, Maudrich M, et al. Universal vaccine against influenza virus: linking TLR signaling to anti-viral protection. Eur J Immunol 2012;42:863-9. doi:10.1002/eji.201041225.

[29] Zhou D, Wu T-L, Lasaro MO, Latimer BP, Parzych EM, Bian A, et al. A universal influenza A vaccine based on adenovirus expressing matrix-2 ectodomain and nucleoprotein protects mice from lethal challenge. Mol Ther 2010;18:2182-9. doi:10.1038/mt.2010.202.

[30] Furuya Y, Chan J, Regner M, Lobigs M, Koskinen A, Kok T, et al. Cytotoxic T cells are the predominant players providing cross-protective immunity induced by $\{$ gamma $\}$-irradiated influenza A viruses. J Virol 2010;84:4212-21. doi:10.1128/JVI.02508-09.

[31] Crevar CJ, Carter DM, Lee KYJ, Ross TM. Cocktail of H5N1 COBRA HA vaccines elicit protective antibodies against H5N1 viruses from multiple clades. Hum Vaccin Immunother 2015. doi:10.1080/21645515.2015.1012013.

[32] Pena L, Sutton T, Chockalingam A, Kumar S, Angel M, Shao H, et al. Influenza viruses with rearranged genomes as live-attenuated vaccines. J Virol 2013;87:5118-27. 
doi:10.1128/JVI.02490-12.

[33] Kallen K-J, Heidenreich R, Schnee M, Petsch B, Schlake T, Thess A, et al. A novel, disruptive vaccination technology. Hum Vaccin Immunother 2014;9:2263-76. doi:10.4161/hv.25181.

[34] Limberis MP, Adam VS, Wong G, Gren J, Kobasa D, Ross TM, et al. Intranasal Antibody Gene Transfer in Mice and Ferrets Elicits Broad Protection Against Pandemic Influenza. Sci Transl Med 2013;5:187ra72-187ra72. doi:10.1126/scitranslmed.3006299.

[35] Kim M-C, Song J-M, O E, Kwon Y-M, Lee Y-J, Compans RW, et al. Virus-like Particles Containing Multiple M2 Extracellular Domains Confer Improved Cross-protection Against Various Subtypes of Influenza Virus. Mol Ther 2012;21:485-92. doi:10.1038/mt.2012.246.

[36] Fu T-M, Grimm KM, Citron MP, Freed DC, Fan J, Keller PM, et al. Comparative immunogenicity evaluations of influenza A virus M2 peptide as recombinant virus like particle or conjugate vaccines in mice and monkeys. Vaccine 2009;27:1440-7. doi:10.1016/j.vaccine.2008.12.034.

[37] Florek NW, Weinfurter JT, Jegaskanda S, Brewoo JN, Powell TD, Young GR, et al. Modified vaccinia virus Ankara encoding influenza virus hemagglutinin induces heterosubtypic immunity in macaques. J Virol 2014;88:13418-28. doi:10.1128/JVI.01219-14.

[38] Yang C, Skiena S, Futcher B, Mueller S, Wimmer E. Deliberate reduction of hemagglutinin and neuraminidase expression of influenza virus leads to an ultraprotective live vaccine in mice. Proc Natl Acad Sci U S A 2013;110:9481-6. doi:10.1073/pnas.1307473110.

[39] Vitelli A, Quirion MR, Lo C-Y, Misplon JA, Grabowska AK, Pierantoni A, et al. Vaccination to Conserved Influenza Antigens in Mice Using a Novel Simian Adenovirus Vector, PanAd3, Derived from the Bonobo Pan paniscus. PLoS One 2013;8:e55435. doi:10.1371/journal.pone.0055435.

[40] De Filette M, Martens W, Roose K, Deroo T, Vervalle F, Bentahir M, et al. An influenza A vaccine based on tetrameric ectodomain of matrix protein 2. J Biol Chem 2008;283:11382-7. doi:10.1074/jbc.M800650200.

[41] Eliasson DG, El Bakkouri K, Schön K, Ramne A, Festjens E, Löwenadler B, et al. CTA1-M2e- 
DD: a novel mucosal adjuvant targeted influenza vaccine. Vaccine 2008;26:1243-52. doi:10.1016/j.vaccine.2007.12.027.

[42] Liu W, Li H, Chen Y-H. N-terminus of M2 protein could induce antibodies with inhibitory activity against influenza virus replication. FEMS Immunol Med Microbiol 2003;35:141-6.

[43] Fan X, Hashem AM, Chen Z, Li C, Doyle T, Zhang Y, et al. Targeting the HA2 subunit of influenza A virus hemagglutinin via CD40L provides universal protection against diverse subtypes. Mucosal Immunol 2015;8:211-20. doi:10.1038/mi.2014.59.

[44] Balazs AB, Bloom JD, Hong CM, Rao DS, Baltimore D. Broad protection against influenza infection by vectored immunoprophylaxis in mice. Nat Biotechnol 2013;31:647-52. doi:10.1038/nbt.2618. 
Table 1. Development Status Of Current Vaccine Candidates

\begin{tabular}{|c|c|c|c|c|c|c|c|}
\hline Organization & Approach, target, adjuvant & 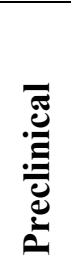 & 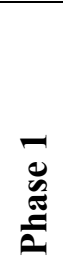 & 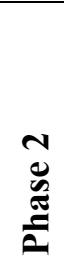 & $\begin{array}{l}m \\
\tilde{E} \\
\tilde{E}\end{array}$ & 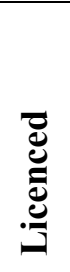 & Reference \\
\hline \multirow[t]{2}{*}{$\begin{array}{l}\text { Novartis } \\
\text { (Switzerland) }\end{array}$} & $\begin{array}{l}\text { Adjuvant MF59 allows for broader cross-reactivity } \\
\text { against viral strains not included in the vaccine. }\end{array}$ & & & & $\mathrm{X}$ & $\mathrm{X}$ & {$[13]$} \\
\hline & $\begin{array}{l}\text { Synthetic, self-amplifying mRNA, delivered by a } \\
\text { synthetic lipid nanoparticle (SAM). }\end{array}$ & $\mathrm{X}$ & & & & & {$[16]$} \\
\hline $\begin{array}{l}\text { GlaxoSmithKline } \\
\text { (UK) }\end{array}$ & $\begin{array}{l}\text { Cross-clade antibody responses demonstrated with split- } \\
\text { virion, inactivated, AS03 adjuvanted vaccine }\end{array}$ & & & & $\mathrm{X}$ & $\mathrm{X}$ & {$[17]$} \\
\hline $\begin{array}{l}\text { Icahn School of } \\
\text { Medicine at Mount } \\
\text { Sinai (USA) and } \\
\text { GlaxoSmithKline } \\
\text { (UK) }\end{array}$ & $\begin{array}{l}\text { Various approaches to target conserved broadly reactive } \\
\text { epitopes on HA stalk, such as 'headless' HA or } \\
\text { functional chimeric HA (comprised of non-matched } \\
\text { 'head' and 'stalk') expressed either in the context of } \\
\text { whole virus or as rHA. }\end{array}$ & $\mathrm{X}$ & & & & & {$[18]$} \\
\hline VaxInnate (USA) & $\begin{array}{l}\text { Fusion protein between influenza M2e and bacterial } \\
\text { flagellin (TLR5 ligand). Self-adjuvanted. Proposed to be } \\
\text { used with conventional trivalent influenza vaccine } \\
\text { (TIV). }\end{array}$ & & & $\mathrm{X}$ & & & {$[11]$} \\
\hline Medicago (Canada) & $\begin{array}{l}\text { Recombinant hemagglutinin (HA) expressed as virus- } \\
\text { like particle (VLP) in tobacco plants. Requires adjuvant. }\end{array}$ & & & $\mathrm{X}$ & & & {$[19]$} \\
\hline $\begin{array}{l}\text { Immune Targeting } \\
\text { Systems (UK) }\end{array}$ & $\begin{array}{l}\text { Six long peptides from four core influenza proteins } \\
\text { conjugated to fluorocarbon chain, elicits strong T cell } \\
\text { response, proposed to be used with conventional TIV. }\end{array}$ & & & $\mathrm{X}$ & & & $\begin{array}{l}\text { http://www.its- } \\
\text { innovation.com }\end{array}$ \\
\hline $\begin{array}{l}\text { BiondVax } \\
\text { Pharmaceuticals } \\
\text { (Israel) }\end{array}$ & $\begin{array}{l}\text { Multimeric- } 001 \text { vaccine: recombinant protein, } \\
\text { combination of nine conserved linear epitopes from HA, } \\
\text { nucleoprotein }(\mathrm{NP}) \text {, and matrix protein }(\mathrm{M}) \text {. }\end{array}$ & & & $\mathrm{X}$ & & & {$[20]$} \\
\hline $\begin{array}{l}\text { SEEK (formerly } \\
\text { PepTcell) (UK) }\end{array}$ & $\begin{array}{l}\text { Flu-V: mixture of four chemically synthesized peptides } \\
\text { targeting conserved T cell epitopes present in M1, NP, } \\
\text { and M2 (with oil-in-water adjuvant) }\end{array}$ & & & $\mathrm{X}$ & & & {$[21]$} \\
\hline $\begin{array}{l}\text { Vivaldi Biosciences } \\
\text { (USAand Austria) }\end{array}$ & $\begin{array}{l}\text { Replication-Deficient Influenza virus created by } \\
\text { deletion of the interferon-inhibiting NS1 protein } \\
\text { activity. }\end{array}$ & & $\mathrm{X}$ & $\mathrm{X}$ & & & {$[22]$} \\
\hline $\begin{array}{l}\text { Acambis Inc. (now } \\
\text { Sanofi) (France) }\end{array}$ & $\begin{array}{l}\text { ACAM-FLU-A Fusion between M2e and hepatitis B } \\
\text { virus core protein (M2e-HBc) to produce VLPs } \\
\text { presenting M2e. }\end{array}$ & & $\mathrm{X}$ & & & & {$[23]$} \\
\hline Inovio (USA) & $\begin{array}{l}\text { DNA plasmids encoding consensus sequences of HA, } \\
\text { NA, and NP delivered by intradermal electroporation for }\end{array}$ & & $\mathrm{X}$ & & & & {$[24]$} \\
\hline
\end{tabular}




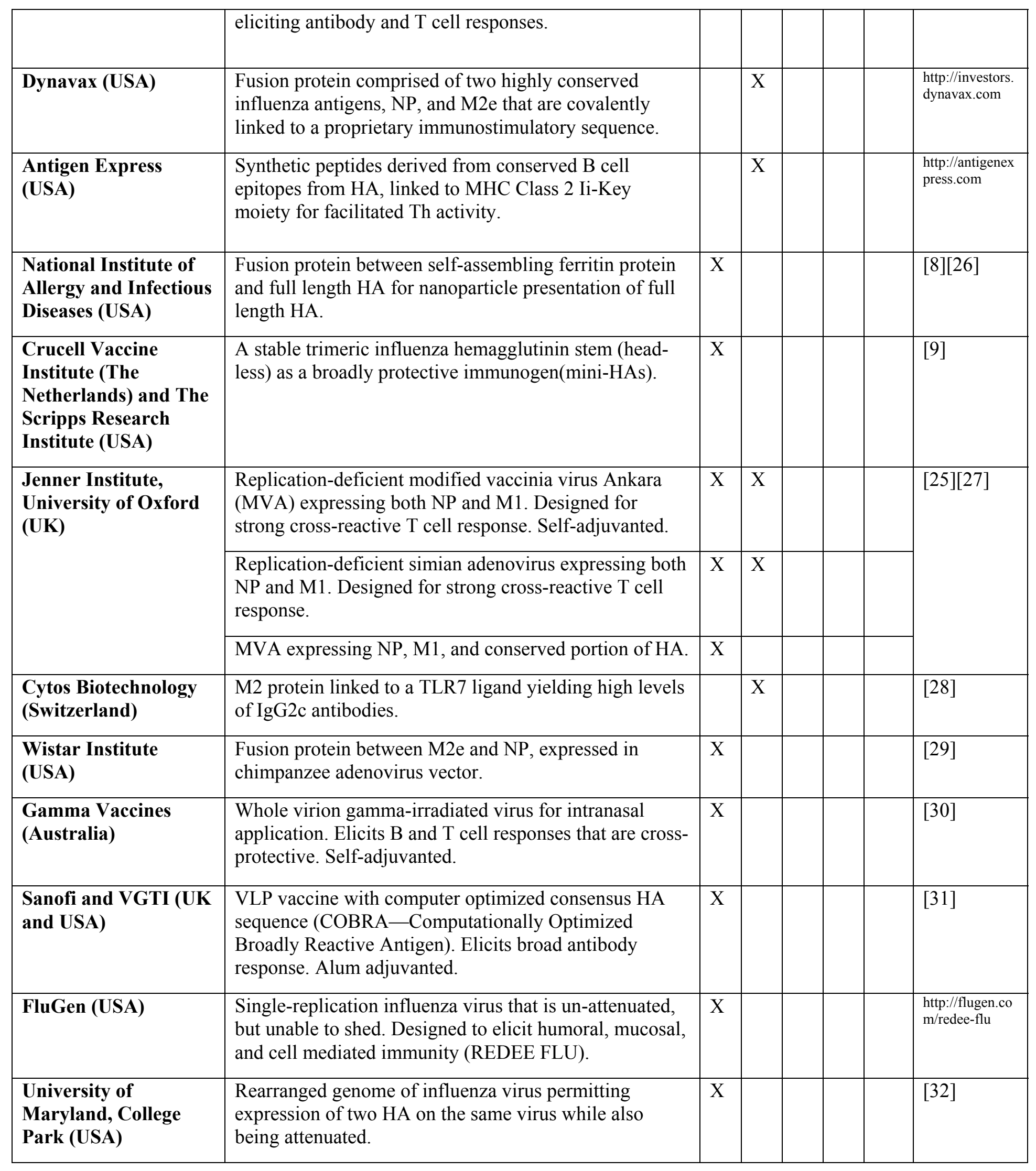




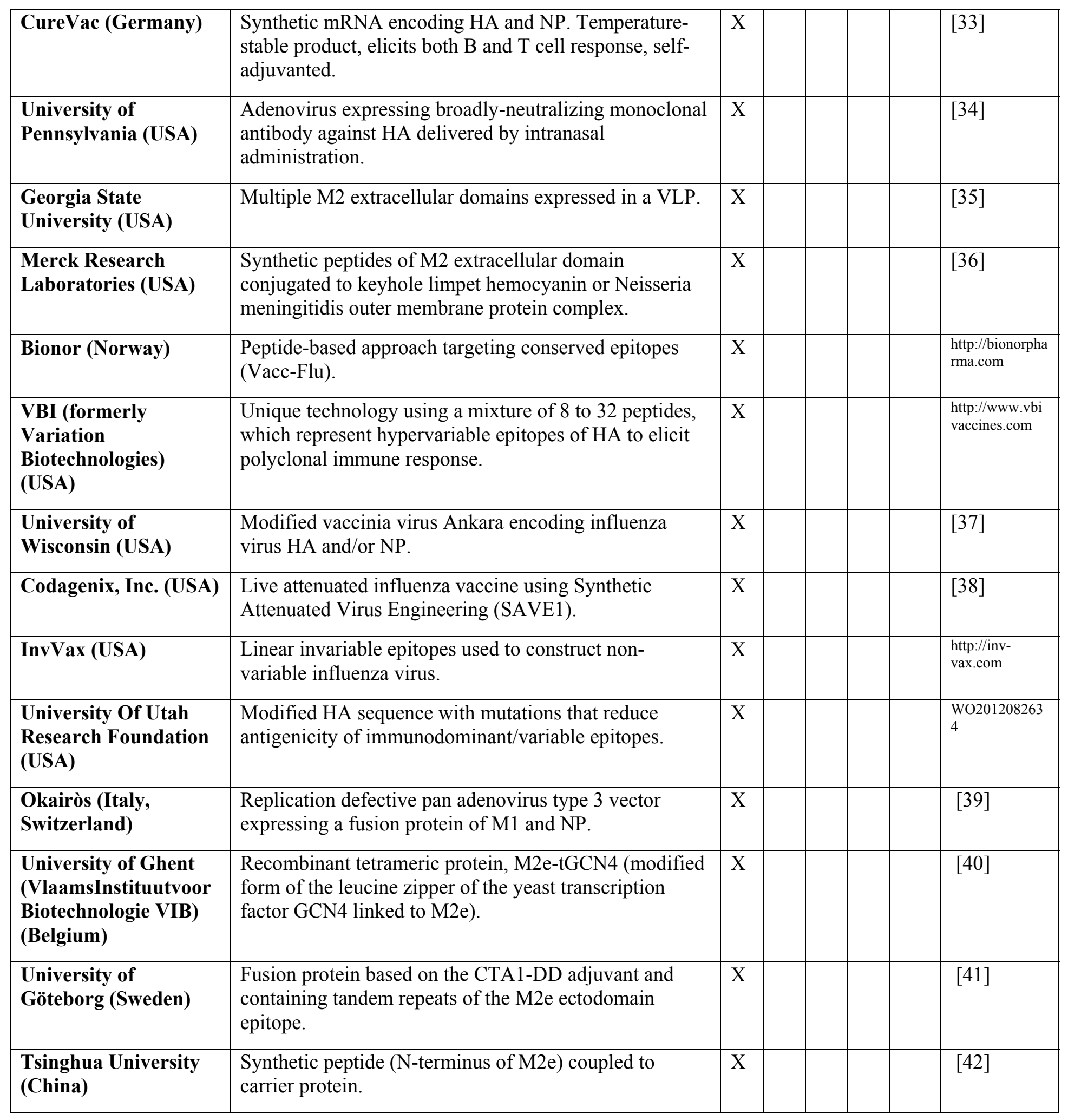




\begin{tabular}{|c|c|c|c|}
\hline $\begin{array}{l}\text { University of Ottawa } \\
\text { (Canada) and } \\
\text { National Institutes for } \\
\text { Food and Drug } \\
\text { Control (China) }\end{array}$ & $\begin{array}{l}\text { Adenovirus vaccine encoding secreted fusion protein } \\
\text { (codon-optimized HA2 subunit fused to a trimerized } \\
\text { form of murine CD40L). }\end{array}$ & $X$ & [43] \\
\hline $\begin{array}{l}\text { California Institute of } \\
\text { Technology (USA) }\end{array}$ & $\begin{array}{l}\text { Adeno-associated viruses delivered intramuscularly } \\
\text { encoding two broadly neutralizing antibodies. }\end{array}$ & $\mathrm{X}$ & [44] \\
\hline
\end{tabular}


Figure 1

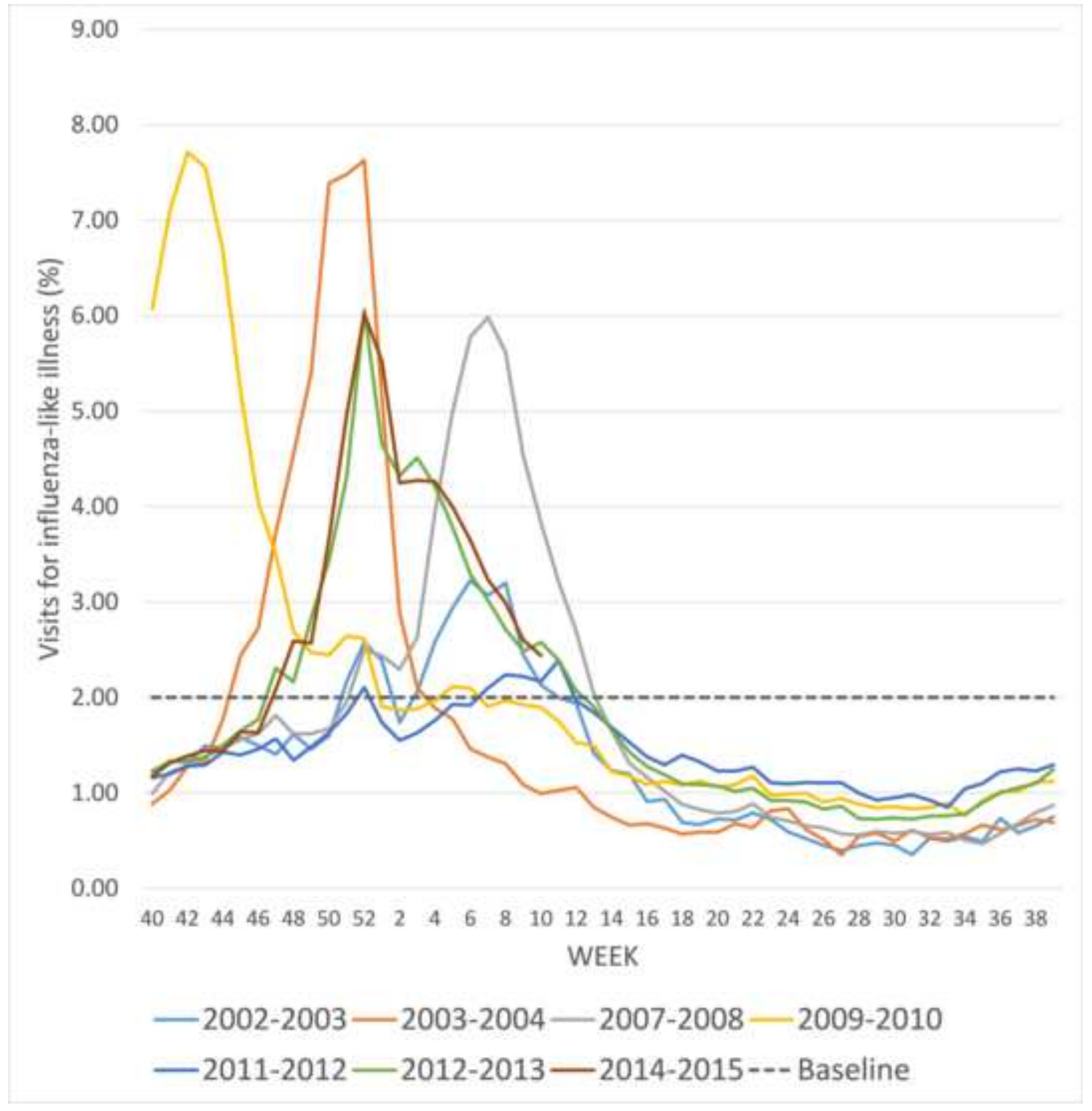

